\title{
A OTIMIZAÇÃO DA SAÚDE DO TRABALHADOR ATRAVÉS DA IMPLANTAÇÃO DA CIF NA MEDICINA DO TRABALHO
}

The optimization of workers' health through the implementation of the ICF in Occupational medicine

Carolina Silva Beltrame, médica graduada pela Universidade do Sul de Santa Catarina; Perita Médica Federal; Especialização em Medicina do Trabalho e em Perícias Médicas; Residência Médica em Pediatria RQE 10133.

E-mail: $\quad$ mdcarolinabeltrame@gmail.com; Endereço para acessar este CV: http://lattes.cnpq.br/8665987889743150.

Joana Silva Beltrame, médica graduada pela Universidade de Caxias do Sul; Especialização em Medicina do Trabalho e Psiquiatria

E-mail: josbeltrame@gmail.com; Endereço para acessar este CV: http://lattes.cnpq.br/1347867599711954

\section{RESUMO:}

Introdução: A Classificação Internacional de Funcionalidade, Incapacidade e Saúde (CIF), faz parte das Classificações Internacionais da Organização Mundial da Saúde juntamente com a CID, sendo publicada em 2001 na 54 Assembleia Mundial de Saúde. Representa uma nova perspectiva, em razão do seu modelo biopsicossocial, classificando o estado de saúde e os fatores que interagem com ela. São avaliadas as alterações nas funções e estruturas do corpo, fatores pessoais, fatores ambientais e, também, barreiras ou facilitadores que interferem na execução de atividades e na participação do indivíduo na sociedade. Objetivo: O objetivo do presente trabalho é propor a criação de um modelo de exame médico ocupacional baseado na CIF, abordando todos os fatores biopsicossociais, através da aplicação de alguns domínios da CIF selecionados pelos autores. O modelo será aplicável no exame admissional, periódico, de retorno ao trabalho, de mudança de função (quando há mudança de risco, conforme a nova NR7) e no exame demissional. Metodologia: O estudo consiste em uma revisão bibliográfica da literatura. Foram consultadas as bases de dados Lilacs, Scielo, Pubmed, as palavras-chave utilizadas foram Classificação Internacional de Funcionalidade, Incapacidade e Saúde, saúde ocupacional e promoção da saúde. Discussão e resultados: Foram encontrados 48 artigos nas bases de dados consultadas. Destes, foram selecionados 15 artigos para compor a bibliografia do trabalho. Baseado nos dados encontrados, criamos um modelo de exame médico ocupacional, selecionando domínios da CIF que se julga ter relação com o labor e/ou que possam influenciar no dia a dia da vida da atividade laborativa. A utilização da CIF permite a inclusão das categorias de atividade, participação e fatores ambientais, o que, de forma superficial, não parecem estar relacionados com à aptidão do trabalhador para a função na empresa. No entanto, quando se identifica nesse trabalhador, dificuldades no âmbito social ou individual e se direciona para uma intervenção precoce, se está, possivelmente, evitando um acidente de trabalho, um afastamento previdenciário prolongado, o absenteísmo, entre outras situações que gerariam um grande ônus para a empresa. Quando se 
implementa a CIF nos exames ocupacionais, se identifica não só as alterações no estado de saúde do trabalhador, como também as características pessoais ou do meio em que vive, podendo restringir sua participação social e no trabalho, fazendo com que altere seu grau de funcionalidade, levando até a uma incapacidade laboral. Considerações finais: A otimização do acompanhamento da saúde do trabalhador com o uso da CIF é um campo desafiador e promissor, que trará benefícios para as três esferas envolvidas: trabalhador, empresa e Serviço Especializado em Segurança e em Medicina do Trabalho (SESMT).

PALAVRAS-CHAVE: Classificação Internacional de Funcionalidade, Incapacidade e Saúde; saúde ocupacional, promoção de saúde.

\section{ABSTRACT:}

Introduction: The International Classification of Functioning, Disability and Health (ICF), is part of the International Classifications of the World Health Organization together with the ICD, being published in 2001 at the 54th World Health Assembly. its biopsychosocial model, classifying the state of health and the factors that interact with it. Changes in body functions and structures, personal factors, environmental factors and also barriers or facilitators that interfere in the performance of activities and in the individual's participation in society are evaluated. Objective: The objective of the study is to propose the creation of an occupational medical examination model based on the ICF, addressing all biopsychosocial factors, through the application of some domains of the ICF selected by the authors. The model will be applicable in the admission exam, periodical, return to work, change of function (when there is a change of risk, according to the new NR7) and in the dismissal exam. Methodology: The study consists of a literature review. Lilacs, Scielo, Pubmed databases were consulted, the keywords used were International Classification of Functioning, Disability and Health, occupational health and health promotion. Discussion and results: 48 articles were found in the consulted databases. Of these, 15 articles were selected to compose the bibliography of this review. Based on the data found, we created an occupational medical examination model, selecting domains of the ICF that were thought to be related to work and/or that could influence the daily life of the work activity. The use of the ICF allows the inclusion of the categories of activity, participation and environmental factors, which, superficially, do not seem to be related to the worker's aptitude for the job in the company. However, when this worker identifies difficulties in the social or individual sphere and directs an early intervention, one is possibly avoiding an accident at work, a prolonged social security absence, absenteeism, among other situations that would generate a great burden for the company. When implementing the ICF in occupational examinations, it identifies not only the changes in the worker's health status, but also the personal characteristics or the environment in which they live, which may restrict their social and work participation, causing them to change their degree of functionality, even leading to incapacity for work. Final considerations: Optimizing worker health monitoring using the ICF is a challenging and promising field, which will bring benefits to the three spheres involved: worker, company and Specialized Service in Safety and Occupational Medicine (SESMT).

KEYWORDS: International Classification of Functioning, Disability and Health; occupational health, health promotion. 


\section{INTRODUÇÃO:}

A Classificação Internacional de Funcionalidade, Incapacidade e Saúde (CIF) foi publicada em 2001, durante a 54a Assembleia Mundial de Saúde, com a finalidade de padronizar uma linguagem internacional de classificação das pessoas com relação ao seu estado de saúde. A CIF faz parte das classificações internacionais da OMS-Organização Mundial da Saúde juntamente com a CID- Classificação Internacional de Doenças e Problemas Relacionados à Saúde ${ }^{1,2,3}$. A CID e a CIF são complementares, no entanto, a CID classifica o estado de saúde das pessoas baseado em um único código alfanumérico, relacionado ao diagnóstico ${ }^{2}$. A CIF, entretanto, permite a classificação considerando o estado e os fatores relacionados à saúde, fazendo uma descrição biopsicossocial do indivíduo, descrevendo todos os aspectos relacionados a funcionalidade e incapacidade das pessoas $^{4}$. O termo funcionalidade engloba todas as funções e estruturas do corpo e atividades e participação, bem como a maneira que indivíduo interage com a sociedade e meio ambiente. $O$ termo incapacidade inclui deficiências, limitações para realizar atividades e restrições na participação. Quando utilizamos a CIF na abordagem das pessoas, conseguimos visualizar o indivíduo como um todo, desde o seu estado de saúde, suas dificuldades, suas limitações como também os fatores que facilitam e influenciam no seu convívio na sociedade ${ }^{1,3,4}$.

O mercado de trabalho é visto como o âmbito em que ocorrem as relações entre indivíduos, instituição e sociedade, além de estar sempre em constante modificação, a qual favorece o dinamismo da sociedade ${ }^{5}$. A constante transformação que o mundo do trabalho sofre, os efeitos da globalização e o avanço da tecnologia, faz com que os trabalhadores também sintam essas mudanças no seu dia a dia e a medicina do trabalho também precisa acompanhar essas mudanças. A maneira como abordar o trabalhador, uma análise mais ampla, que consiga verificar as dificuldades e limitações dos trabalhadores é muito importante ${ }^{5}$.

$\mathrm{Na}$ medicina do trabalho, de acordo com a Norma Regulamentadora número 7 (NR 7), os exames médicos ocupacionais são de realização obrigatória e estão incluídos no Programa de Controle Médico e Saúde Ocupacional (PCMSO), fazendo parte das atividades do Serviço Especializado em Segurança e Medicina do Trabalho (SESMT). São eles: admissional, periódico, de retorno ao trabalho, mudança de função (quando há mudança de fator de risco) e demissional. Esses exames são de suma importância dentro da saúde ocupacional pois através deles avaliamos condições de saúde do trabalhador para determinada(s) atividade(s), estabelecendo o perfil de saúde desses trabalhadores, detectando alterações préclínicas e clínicas, proporcionando que se adotem medidas na prevenção e no agravo da saúde, bem como medidas de promoção da saúde, e assim fortalecendo métodos de trabalho seguros e manutenção da saúde ${ }^{6}$.

De acordo com dados da Organização Internacional do Trabalho (OIT), ocorrem anualmente 300 milhões de acidentes de trabalho em todo o mundo. No Brasil, cerca de 700 mil casos são registrados em média todos os anos, fazendo com que o país gaste $R \$ 70$ bilhões com acidentes de trabalho anualmente. Nesse sentido, é necessário cada vez mais, que os exames médicos ocupacionais sejam de excelente qualidade e, com o uso da CIF, é possível categorizar todas as informações necessárias sobre o trabalhador para que o médico possa agir precocemente, identificando as dificuldades desse trabalhador e prevenindo a ocorrência de acidentes de trabalho e o afastamento previdenciário ${ }^{7}$.

\section{OBJETIVO:}

O objetivo do presente trabalho é propor a criação de um modelo de exame 
médico ocupacional baseado na CIF, abordando todos os fatores biopsicossociais, através da aplicação de categorias e domínios da CIF selecionados pelos autores. O modelo será aplicável no exame admissional, periódico, de retorno ao trabalho, de mudança de função (quando há mudança de risco, conforme a nova NR7) e no exame demissional.

\section{METODOLOGIA:}

O estudo consiste em uma revisão bibliográfica da literatura. Foram consultadas as bases de dados Lilacs, Scielo, Pubmed. As palavras-chave utilizadas foram Classificação Internacional de Funcionalidade, Incapacidade e Saúde, saúde do trabalhador, promoção de saúde. Com base nessa revisão, criou-se um modelo de exame médico ocupacional utilizando os domínios da CIF, aplicáveis nos exames admissional, periódicos, de mudança de função (quando há mudança de fator de risco), de retorno ao trabalho e demissional.

\section{DISCUSSÃO E RESULTADOS:}

Foram encontrados 48 artigos nas bases de dados consultadas. Destes, foram selecionados 15 artigos para compor a bibliografia do trabalho. Baseado nos dados achados, criamos um modelo de exame médico ocupacional, selecionando domínios da CIF que se julga ter relação com o labor e/ou que possam influenciar no dia a dia da vida laborativa.

Primeiramente, se coleta os dados pessoais: nome completo, data de nascimento, escolaridade, como habitualmente se realiza em todas as anamneses. Identifica-se 0 cargo a ser executado e atividades relacionadas ao cargo. Nesse item, propõe-se ao médico que identifique três atividades que o empregado desenvolverá no cargo pretendido e que verifique se ele está apto para a função. Por exemplo, um motorista, realizará atividades de dirigir (d475), necessita permanecer longos períodos na posição sentada, (d415) e necessita ter boa acuidade visual (b210).

Em seguida, se avalia as atividades e participação do trabalhador, bem como os fatores ambientais aos quais ele está exposto. Atividade é a execução de uma tarefa por um indivíduo. Participação é o envolvimento do indivíduo numa situação da vida real, como, por exemplo, no ambiente de trabalho. Quando há limitações na atividade, o indivíduo possui dificuldades na execução. Quando há restrições na participação, o indivíduo pode enfrentar problemas em situações da vida real $^{1,3}$. O qualificador de desempenho descreve o que o indivíduo faz no seu ambiente habitual, o seu envolvimento numa situação real da vida. No item: aprendizagem e aplicação dos conhecimentos, foram selecionados alguns domínios da CIF, como adquirir competências, concentrar a atenção, resolver problemas, tomar decisões e aplicação do conhecimento. Tarefas e exigências gerais trata dos aspectos gerais da execução de uma única ou várias tarefas, organização de rotinas e gestão do estresse. $\mathrm{Na}$ comunicação avalia-se características gerais e específicas da comunicação através da linguagem incluindo a recepção e a produção de mensagens e utilização de dispositivos e técnicas de comunicação. Consecutivamente, nos demais domínios de mobilidade, autocuidado, vida doméstica, interações e relacionamentos interpessoais, principais áreas da vida, vida comunitária, social e cívica foram selecionados domínios da CIF que se relacionam com o ambiente de trabalho e fatores que podem influenciar no desempenho e funcionalidade do trabalhador.

Para se qualificar o grau de deficiência ou as limitações graduamos em nenhuma deficiência (0-4\%), deficiência leve (5-24\%), moderada (25-49\%), grave (50 a $95 \%$ ) e total ou completa (96 a 100\%). Utiliza-se o qualificador 8- não especificado, quando as informações são insuficientes para permitir uma designação de uma categoria mais específica ${ }^{1,3}$. 
FIGURA 1: AVALIAÇÃO DA ATIVIDADE E PARTICIPAÇÃO

\begin{tabular}{|l|c|c|c|c|c|}
\hline \multicolumn{5}{|c|}{ Aprendizagem e aplicação dos conhecimentos (d1) } \\
\hline & $\begin{array}{c}\text { 0- sem } \\
\text { dificuldades }\end{array}$ & $\begin{array}{c}\text { 1- LEVE } \\
\text { dificuldade }\end{array}$ & $\begin{array}{c}\text { 2- } \\
\text { MODERADA } \\
\text { dificuldade }\end{array}$ & $\begin{array}{l}\text { 3- GRAVE } \\
\text { dificuldade }\end{array}$ & $\begin{array}{l}\text { 4- TOTAL } \\
\text { dificuldade }\end{array}$ \\
\hline $\begin{array}{l}\text { d155 adquirir } \\
\text { competências }\end{array}$ & $\bigcirc$ & $\bigcirc$ & $\bigcirc$ & $\bigcirc$ & $\bigcirc$ \\
\hline $\begin{array}{l}\text { d160 concentrar } \\
\text { a atenção }\end{array}$ & $\bigcirc$ & $\bigcirc$ & $\bigcirc$ & $\bigcirc$ & $\bigcirc$ \\
\hline $\begin{array}{l}\text { d175 resolver } \\
\text { problemas }\end{array}$ & $\bigcirc$ & $\bigcirc$ & $\bigcirc$ & $\bigcirc$ & $\bigcirc$ \\
\hline $\begin{array}{l}\text { d177 tomar } \\
\text { decisões }\end{array}$ & $\bigcirc$ & $\bigcirc$ & $\bigcirc$ & $\bigcirc$ & $\bigcirc$ \\
\hline $\begin{array}{l}\text { d179 aplicação } \\
\text { do } \\
\text { conhecimento }\end{array}$ & $\bigcirc$ & $\bigcirc$ & $\bigcirc$ & $\bigcirc$ & $\bigcirc$ \\
\hline
\end{tabular}

\begin{tabular}{|l|c|c|c|c|c|}
\hline \multicolumn{7}{|c|}{ Tarefas e exigências gerais (d2) } \\
\hline & $\begin{array}{c}\text { 0- sem } \\
\text { dificuldades }\end{array}$ & $\begin{array}{c}\text { 1- LEVE } \\
\text { dificuldade }\end{array}$ & $\begin{array}{c}\text { 2- } \\
\text { MODERADA } \\
\text { dificuldade }\end{array}$ & $\begin{array}{l}\text { 3- GRAVE } \\
\text { dificuldade }\end{array}$ & $\begin{array}{l}\text { 4- TOTAL } \\
\text { dificuldade }\end{array}$ \\
\hline $\begin{array}{l}\text { d220 realizar } \\
\text { tarefas múltiplas }\end{array}$ & $\bigcirc$ & $\bigcirc$ & $\bigcirc$ & $\bigcirc$ & $\bigcirc$ \\
\hline $\begin{array}{l}\text { d230 gerir sua } \\
\text { rotina diária }\end{array}$ & $\bigcirc$ & $\bigcirc$ & $\bigcirc$ & $\bigcirc$ & $\bigcirc$ \\
\hline $\begin{array}{l}\text { d240 lidar com o } \\
\text { estresse ou } \\
\text { outras } \\
\text { exigências } \\
\text { psicológicas }\end{array}$ & $\bigcirc$ & $\bigcirc$ & $\bigcirc$ & $\bigcirc$ & $\bigcirc$ \\
\hline
\end{tabular}

\begin{tabular}{|l|c|c|c|c|c|}
\hline \multicolumn{5}{|c|}{ Comunicação (d3) } \\
\hline & $\begin{array}{l}\text { 0- sem } \\
\text { dificuldades }\end{array}$ & $\begin{array}{c}\text { 1- LEVE } \\
\text { dificuldade }\end{array}$ & $\begin{array}{c}\text { 2- } \\
\text { MODERADA } \\
\text { dificuldade }\end{array}$ & $\begin{array}{l}\text { 3- GRAVE } \\
\text { dificuldade }\end{array}$ & $\begin{array}{c}\text { 4- TOTAL } \\
\text { dificuldade }\end{array}$ \\
\hline $\begin{array}{l}\text { d310 comunicar } \\
\text { e receber } \\
\text { mensagens } \\
\text { orais }\end{array}$ & $\bigcirc$ & 0 & 0 & 0 & $\bigcirc$ \\
\hline $\begin{array}{l}\text { d360 utilização } \\
\text { de dispositivos e } \\
\text { técnicas de } \\
\text { comunicação }\end{array}$ & $\bigcirc$ & $\bigcirc$ & $\bigcirc$ & $\bigcirc$ & $\bigcirc$ \\
\hline
\end{tabular}




\begin{tabular}{|l|c|c|c|c|c|}
\hline \multicolumn{7}{|c|}{ Mobilidade (d4) } \\
\hline & $\begin{array}{c}\text { 0- sem } \\
\text { dificuldades }\end{array}$ & $\begin{array}{c}\text { 1- LEVE } \\
\text { dificuldade }\end{array}$ & $\begin{array}{c}\text { 2- } \\
\text { MODERADA } \\
\text { dificuldade }\end{array}$ & $\begin{array}{l}\text { 3- GRAVE } \\
\text { dificuldade }\end{array}$ & $\begin{array}{l}\text { 4- TOTAL } \\
\text { dificuldade }\end{array}$ \\
\hline $\begin{array}{l}\text { d410 mudar as } \\
\text { posições do } \\
\text { corpo }\end{array}$ & $\bigcirc$ & $\bigcirc$ & $\bigcirc$ & $\bigcirc$ & $\bigcirc$ \\
$\begin{array}{l}\text { d415 manter a } \\
\text { posição do } \\
\text { corpo }\end{array}$ & $\bigcirc$ & $\bigcirc$ & $\bigcirc$ & $\bigcirc$ & $\bigcirc$ \\
\hline $\begin{array}{l}\text { d430 levantar e } \\
\text { transportar } \\
\text { objetos }\end{array}$ & $\bigcirc$ & $\bigcirc$ & $\bigcirc$ & $\bigcirc$ & $\bigcirc$ \\
\hline $\begin{array}{l}\text { d445 utilização } \\
\text { de mão e braços }\end{array}$ & $\bigcirc$ & $\bigcirc$ & $\bigcirc$ & $\bigcirc$ & $\bigcirc$ \\
\hline $\begin{array}{l}\text { d470 utilizar um } \\
\text { transporte }\end{array}$ & $\bigcirc$ & $\bigcirc$ & $\bigcirc$ & $\bigcirc$ & $\bigcirc$ \\
\hline $\begin{array}{l}\text { d475 } \\
\text { conduzir/dirigir }\end{array}$ & $\bigcirc$ & $\bigcirc$ & $\bigcirc$ & $\bigcirc$ & $\bigcirc$ \\
\hline
\end{tabular}

\begin{tabular}{|l|c|c|c|c|c|}
\hline \multicolumn{7}{|c|}{ Autocuidados (d5) } \\
\hline & $\begin{array}{c}\text { 0- sem } \\
\text { dificuldades }\end{array}$ & $\begin{array}{c}\text { 1- LEVE } \\
\text { dificuldade }\end{array}$ & $\begin{array}{c}\text { 2- } \\
\text { MODERADA } \\
\text { dificuldade }\end{array}$ & $\begin{array}{l}\text { 3- GRAVE } \\
\text { dificuldade }\end{array}$ & $\begin{array}{c}\text { 4- TOTAL } \\
\text { dificuldade }\end{array}$ \\
\hline $\begin{array}{l}\text { d520 cuidar de } \\
\text { partes do corpo }\end{array}$ & $\bigcirc$ & $\bigcirc$ & $\bigcirc$ & $\bigcirc$ & $\bigcirc$ \\
\hline $\begin{array}{l}\text { d570 cuidar da } \\
\text { própria saúde }\end{array}$ & $\bigcirc$ & $\bigcirc$ & $\bigcirc$ & $\bigcirc$ & $\bigcirc$ \\
\hline
\end{tabular}

\begin{tabular}{|l|c|c|c|l|c|}
\hline \multicolumn{7}{|c|}{ Vida doméstica (d6) } \\
\hline & $\begin{array}{c}\text { 0- sem } \\
\text { dificuldades }\end{array}$ & $\begin{array}{c}\text { 1- LEVE } \\
\text { dificuldade }\end{array}$ & $\begin{array}{c}\text { 2- } \\
\text { MODERADA } \\
\text { dificuldade }\end{array}$ & $\begin{array}{l}\text { 3- } \\
\text { GRAVE } \\
\text { dificuldade }\end{array}$ & $\begin{array}{c}\text { 4- TOTAL } \\
\text { dificuldade }\end{array}$ \\
\hline $\begin{array}{l}\text { d640 realizar o } \\
\text { trabalho } \\
\text { doméstico }\end{array}$ & $\bigcirc$ & $\bigcirc$ & $\bigcirc$ & $\bigcirc$ & $\bigcirc$ \\
\hline
\end{tabular}

\begin{tabular}{|l|c|c|c|c|c|}
\hline \multicolumn{7}{|c|}{ Interações e relacionamentos interpessoais (d7) } \\
\hline & $\begin{array}{c}\text { 0- sem } \\
\text { dificuldades }\end{array}$ & $\begin{array}{c}\text { 1- LEVE } \\
\text { dificuldade }\end{array}$ & $\begin{array}{c}\text { 2- } \\
\text { MODERADA } \\
\text { dificuldade }\end{array}$ & $\begin{array}{l}\text { 3- GRAVE } \\
\text { dificuldade }\end{array}$ & $\begin{array}{c}\text { 4- TOTAL } \\
\text { dificuldade }\end{array}$ \\
\hline $\begin{array}{l}\text { d710 interações } \\
\text { interpessoais } \\
\text { básicas }\end{array}$ & $\bigcirc$ & $\bigcirc$ & $\bigcirc$ & $\bigcirc$ & $\bigcirc$ \\
\hline $\begin{array}{l}\text { d740 } \\
\text { relacionamento } \\
\text { formais (com }\end{array}$ & $\bigcirc$ & $\bigcirc$ & $\bigcirc$ & $\bigcirc$ & $\bigcirc$ \\
\hline
\end{tabular}




\begin{tabular}{|l|c|c|c|c|c|}
\hline $\begin{array}{l}\text { superiores, } \\
\text { subordinados) }\end{array}$ & & & & & \\
\hline $\begin{array}{l}\text { d750 } \\
\text { relacionamentos } \\
\text { sociais informais }\end{array}$ & $\bigcirc$ & $\bigcirc$ & $\bigcirc$ & $\bigcirc$ & $\bigcirc$ \\
\hline $\begin{array}{l}\text { d760 } \\
\text { relacionamentos } \\
\text { familiares }\end{array}$ & $\bigcirc$ & $\bigcirc$ & $\bigcirc$ & $\bigcirc$ & $\bigcirc$ \\
\hline
\end{tabular}

\begin{tabular}{|l|c|c|c|c|c|}
\hline \multicolumn{7}{|c|}{ Principais áreas da vida (d8) } \\
\hline & $\begin{array}{c}\text { 0- sem } \\
\text { dificuldades }\end{array}$ & $\begin{array}{c}\text { 1- LEVE } \\
\text { dificuldade }\end{array}$ & $\begin{array}{c}\text { 2- } \\
\text { MODERADA } \\
\text { dificuldade }\end{array}$ & $\begin{array}{l}\text { 3- GRAVE } \\
\text { dificuldade }\end{array}$ & $\begin{array}{l}\text { 4- TOTAL } \\
\text { dificuldade }\end{array}$ \\
\hline $\begin{array}{l}\text { d825 } \\
\text { treinamento } \\
\text { profissional }\end{array}$ & $\bigcirc$ & $\bigcirc$ & $\bigcirc$ & $\bigcirc$ & $\bigcirc$ \\
\hline $\begin{array}{l}\text { d845 conseguir, } \\
\text { manter e sair do } \\
\text { emprego }\end{array}$ & $\bigcirc$ & $\bigcirc$ & $\bigcirc$ & $\bigcirc$ & $\bigcirc$ \\
\hline $\begin{array}{l}\text { d870 } \\
\text { autossuficiência } \\
\text { econômica }\end{array}$ & $\bigcirc$ & $\bigcirc$ & $\bigcirc$ & $\bigcirc$ & $\bigcirc$ \\
\hline
\end{tabular}

\begin{tabular}{|l|c|c|c|c|c|}
\hline \multicolumn{7}{|c|}{ Vida comunitária, social e cívica (d9) } \\
\hline & $\begin{array}{c}\text { 1- - sem } \\
\text { dificuldades }\end{array}$ & $\begin{array}{c}\text { 2- } \\
\text { dificuldade }\end{array}$ & $\begin{array}{c}\text { MODERADA } \\
\text { dificuldade }\end{array}$ & $\begin{array}{l}\text { 3- GRAVE } \\
\text { dificuldade }\end{array}$ & $\begin{array}{l}\text { 4- TOTAL } \\
\text { dificuldade }\end{array}$ \\
\hline $\begin{array}{l}\text { d910 vida } \\
\text { comunitária }\end{array}$ & $\bigcirc$ & $\bigcirc$ & $\bigcirc$ & $\bigcirc$ & $\bigcirc$ \\
\hline $\begin{array}{l}\text { d920 recreação } \\
\text { e lazer }\end{array}$ & $\bigcirc$ & $\bigcirc$ & $\bigcirc$ & $\bigcirc$ & $\bigcirc$ \\
\hline $\begin{array}{l}\text { d930 religião e } \\
\text { espiritualidade }\end{array}$ & $\bigcirc$ & $\bigcirc$ & $\bigcirc$ & $\bigcirc$ & $\bigcirc$ \\
\hline
\end{tabular}

Observações com relação as respostas dos itens de atividades e participação - d1 a d9.

Os fatores ambientais, do quadro a seguir, constituem o ambiente físico, social e atitudinal em que as pessoas vivem e conduzem a sua vida ${ }^{1,3}$. No caso da medicina do trabalho, selecionou-se dois itens: medicamentos (se a pessoa faz uso de alguma medicação) e produtos e tecnologias de apoio para uso pessoal na vida diária (incluiu as órteses, próteses, cadeira de rodas, bengala que a pessoa necessita utilizar no seu dia a dia). Diferentes ambientes podem ter impacto distinto sobre o mesmo indivíduo com determinada condição de saúde. Um ambiente com barreiras pode restringir $o$ desempenho do indivíduo, enquanto ambientes com facilitadores podem melhorar 
esses desempenho. Um exemplo são prédios sem rampa de acesso ou sem elevadores que constituem uma barreira para o trabalhador ${ }^{1,3}$.

\section{FIGURA 2: AVALIAÇÃO DOS FATORES AMBIENTAIS}

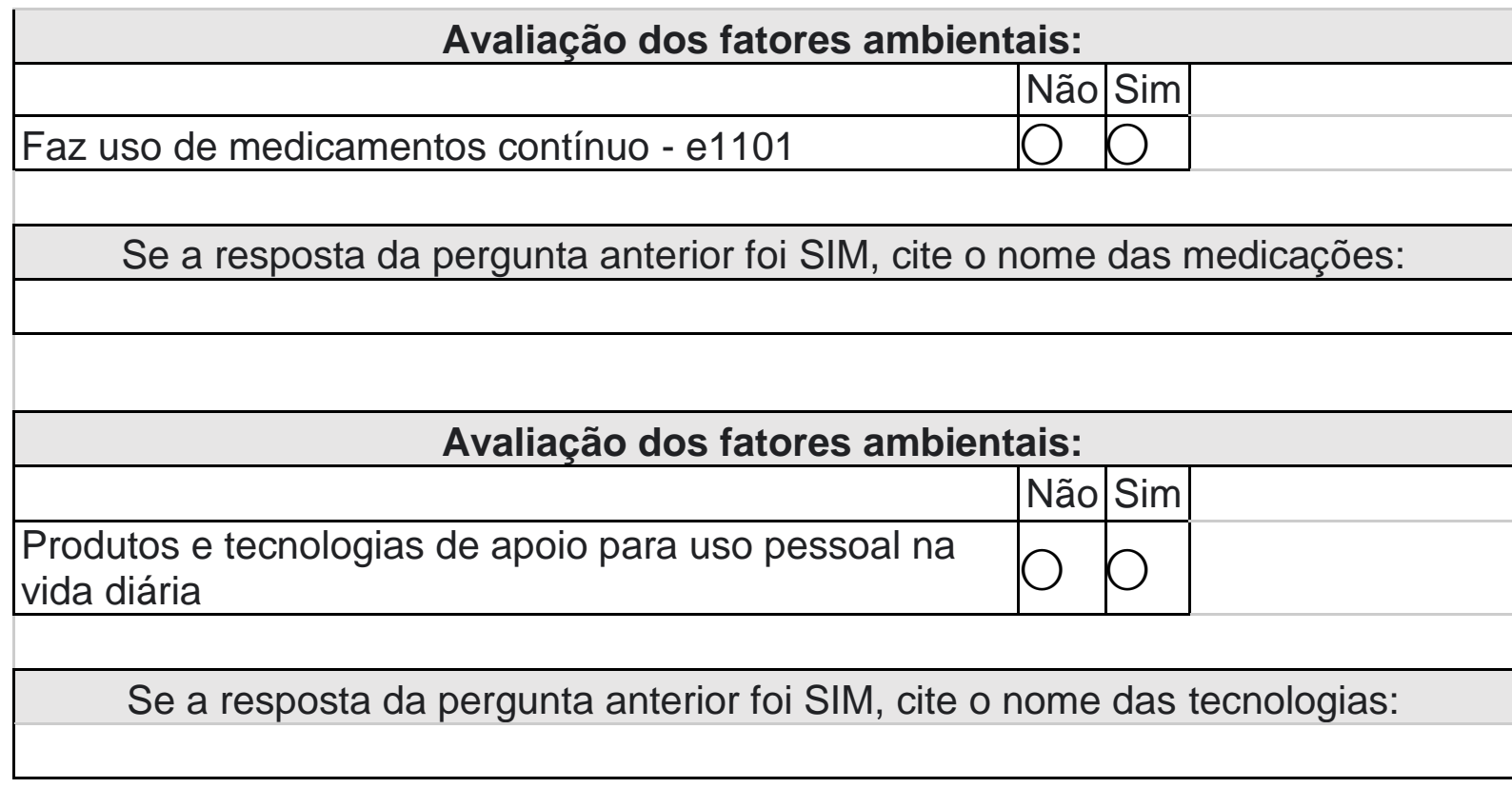

\section{Fonte: os autores}

Ao se avaliar as funções do corpo, se está avaliando as funções fisiológicas dos sistemas orgânicos (incluindo as funções psicológicas). As estruturas do corpo são as partes anatômicas. Pode haver deficiências, que são problemas nas funções ou estruturas do corpo, tais como, um desvio importante ou uma perda ${ }^{1,3}$.

FIGURA 3: AVALIAÇÃO DAS FUNÇÕES E ESTRUTURAS DO CORPO

$$
\text { Avaliação das estruturas e funções do corpo }
$$

\begin{tabular}{|l|c|c|c|c|c|c|}
\hline \multicolumn{7}{|c|}{ Possui alterações nas funções do corpo? } \\
\hline & $\begin{array}{c}\text { Não } \\
\text { Leve } \\
-1\end{array}$ & $\begin{array}{c}\text { Sim.Moderada } \\
-2\end{array}$ & $\begin{array}{c}\text { Sim.Grave } \\
-3\end{array}$ & $\begin{array}{c}\text { Sim. Total } \\
-4\end{array}$ & $\begin{array}{c}\text { Sim. Não } \\
\text { especificada } \\
-8\end{array}$ \\
\hline Funções mentais & 0 & 0 & $\bigcirc$ & $\bigcirc$ & $\bigcirc$ & $\bigcirc$ \\
\hline
\end{tabular}

Caso a resposta anterior tenha sido SIM, especifique: 


\begin{tabular}{|l|c|c|c|c|c|c|}
\hline \multicolumn{7}{|c|}{ Possui alterações nas funções do corpo? } \\
\hline & $\begin{array}{c}\text { Não } \\
\text { Leve } \\
-1\end{array}$ & $\begin{array}{c}\text { Sim.Moderada } \\
-2\end{array}$ & $\begin{array}{c}\text { Sim.Grave } \\
-3\end{array}$ & $\begin{array}{c}\text { Sim.Total } \\
-4\end{array}$ & $\begin{array}{c}\text { Sim. Não } \\
\text { especificada } \\
-8\end{array}$ \\
\hline $\begin{array}{l}\text { Funções } \\
\text { sensoriais e dor }\end{array}$ & $\bigcirc$ & $\bigcirc$ & $\bigcirc$ & $\bigcirc$ & $\bigcirc$ & $\bigcirc$ \\
\hline
\end{tabular}

Caso a resposta anterior tenha sido SIM, especifique:

\begin{tabular}{|l|c|c|c|c|c|c|}
\hline \multicolumn{7}{|c|}{ Possui alterações nas funções do corpo? } \\
\hline & $\begin{array}{c}\text { Não } \\
\text { Lim. } \\
\text { Leve } \\
-1\end{array}$ & $\begin{array}{c}\text { Sim.Moderada } \\
-2\end{array}$ & $\begin{array}{c}\text { Sim.Grave } \\
-3\end{array}$ & $\begin{array}{c}\text { Sim.Total } \\
-4\end{array}$ & $\begin{array}{c}\text { Sim. Não } \\
\text { especificada } \\
-8\end{array}$ \\
\hline $\begin{array}{l}\text { Funções da voz } \\
\text { e da fala }\end{array}$ & $\bigcirc$ & $\bigcirc$ & $\bigcirc$ & $\bigcirc$ & $\bigcirc$ & $\bigcirc$ \\
\hline
\end{tabular}

Caso a resposta anterior tenha sido SIM, especifique:

\begin{tabular}{|l|c|c|c|c|c|c|}
\hline \multicolumn{6}{|c|}{ Possui alterações nas funções do corpo? } \\
\hline & $\begin{array}{r}\text { Não } \\
\text { Leve } \\
-1\end{array}$ & $\begin{array}{c}\text { Sim.Moderada } \\
-2\end{array}$ & $\begin{array}{c}\text { Sim.Grave } \\
-3\end{array}$ & $\begin{array}{c}\text { Sim.Total } \\
-4\end{array}$ & $\begin{array}{c}\text { Sim. Não } \\
\text { especificada } \\
-8\end{array}$ \\
\hline $\begin{array}{l}\text { Funções do } \\
\text { aparelho } \\
\text { cardiovascular, } \\
\text { dos sistemas } \\
\text { hematológico e } \\
\text { imunológico e do } \\
\begin{array}{l}\text { aparelho } \\
\text { respiratório }\end{array}\end{array}$ & $\bigcirc$ & $\bigcirc$ & 0 & 0 & $\bigcirc$ & $\bigcirc$ \\
\hline
\end{tabular}

Caso a resposta anterior tenha sido SIM, especifique:

\begin{tabular}{|l|c|c|c|c|c|c|}
\hline \multicolumn{7}{|c|}{ Possui alterações nas funções do corpo? } \\
\hline & $\begin{array}{r}\text { Não } \\
\text { Leve } \\
\text { Leve }\end{array}$ & $\begin{array}{c}\text { Sim.Moderada } \\
-2\end{array}$ & $\begin{array}{c}\text { Sim.Grave } \\
-3\end{array}$ & $\begin{array}{c}\text { Sim.Total } \\
-4\end{array}$ & $\begin{array}{c}\text { Sim. Não } \\
\text { especificada } \\
-8\end{array}$ \\
\hline $\begin{array}{l}\text { Funções do } \\
\text { aparelho } \\
\text { digestivo e dos } \\
\text { sistemas } \\
\text { metabólico e } \\
\text { endócrino }\end{array}$ & $\wp$ & $\bigcirc$ & 0 & 0 & $\bigcirc$ & $\bigcirc$ \\
\hline
\end{tabular}

Caso a resposta anterior tenha sido SIM, especifique: 


\begin{tabular}{|l|c|c|c|c|c|c|}
\hline \multicolumn{7}{|c|}{ Possui alterações nas funções do corpo? } \\
\hline & Não & $\begin{array}{c}\text { Sim. } \\
\text { Leve } \\
-1\end{array}$ & $\begin{array}{c}\text { Sim.Moderada } \\
-2\end{array}$ & $\begin{array}{c}\text { Sim.Grave } \\
-3\end{array}$ & $\begin{array}{c}\text { Sim. Total } \\
-4\end{array}$ & $\begin{array}{c}\text { Sim. Não } \\
\text { especificada } \\
-8\end{array}$ \\
\hline $\begin{array}{l}\text { Funções } \\
\text { geniturinárias e } \\
\text { reprodutivas }\end{array}$ & $\bigcirc$ & $\bigcirc$ & 0 & 0 & 0 & $\bigcirc$ \\
\hline
\end{tabular}

Caso a resposta anterior tenha sido SIM, especifique:

\begin{tabular}{|l|c|c|c|c|c|c|}
\hline \multicolumn{7}{|c|}{ Possui alterações nas funções do corpo? } \\
\hline & $\begin{array}{c}\text { Não } \\
\text { Lim. } \\
\text { Leve } \\
-1\end{array}$ & $\begin{array}{c}\text { Sim.Moderada } \\
-2\end{array}$ & $\begin{array}{c}\text { Sim.Grave } \\
-3\end{array}$ & $\begin{array}{c}\text { Sim. Total } \\
-4\end{array}$ & $\begin{array}{c}\text { Sim. Não } \\
\text { especificada } \\
-8\end{array}$ \\
\hline $\begin{array}{l}\text { Funções } \\
\text { neuromusculares } \\
\text { esqueléticas e } \\
\text { relacionadas } \\
\text { com os } \\
\text { movimentos }\end{array}$ & $\bigcirc$ & $\bigcirc$ & 0 & 0 & 0 & $\bigcirc$ \\
\hline
\end{tabular}

Caso a resposta anterior tenha sido SIM, especifique:

\begin{tabular}{|l|c|c|c|c|c|c|}
\hline \multicolumn{7}{|c|}{ Possui alterações nas funços do corpo? } \\
\hline & Não & $\begin{array}{c}\text { Sim. } \\
\text { Leve } \\
-1\end{array}$ & $\begin{array}{c}\text { Sim.Moderada } \\
-2\end{array}$ & $\begin{array}{c}\text { Sim.Grave } \\
-3\end{array}$ & $\begin{array}{c}\text { Sim. Total } \\
-4\end{array}$ & $\begin{array}{c}\text { Sim. Não } \\
\text { especificada } \\
-8\end{array}$ \\
\hline $\begin{array}{l}\text { Funções da pele } \\
\text { e estruturas } \\
\text { relacionadas }\end{array}$ & $\bigcirc$ & $\bigcirc$ & 0 & 0 & 0 & $\bigcirc$ \\
\hline
\end{tabular}

Caso a resposta anterior tenha sido SIM, especifique:

Enquadra-se nos critérios de Pessoas com Deficiência? De acordo com o artigo $2^{\circ}$ da Lei $n^{\circ} 13.146 / 2015$; dos artigos $3^{\circ}$ e $4^{\circ}$ do Decreto $n^{\circ} 3.298 / 1999$, com as alterações do Decreto $n^{\circ} 5.296 / 2004$; do artigo $1^{\circ}, \S^{\circ}$ da Lei $n^{\circ}$

12.764/2012, Parecer CONJUR $n^{\circ} 444 / 2011$, das recomendações da IN $n^{\circ}$ 98/SIT/2012 e de acordo com dispositivos relativos do Decreto $n^{\circ} 6.949 / 2009$.

\begin{tabular}{|l|l|l|}
\hline & Sim & \\
\cline { 1 - 1 } & Não & \\
\hline
\end{tabular}


Dentre os exames ocupacionais, o exame admissional é realizado antes da inserção do trabalhador na empresa, a coleta desses dados baseados na CIF serve como referência para 0 acompanhamento do trabalhador, além do objetivo principal do exame que é a conclusão de aptidão para a função. Muitos termos já são utilizados ao realizar esses exames, como capacidade/incapacidade, funcionalidade, aptidão, desempenho, porém os profissionais envolvidos não têm a exata dimensão de seus significados e o quanto esses registros podem facilitar a adoção de medidas e controle dos trabalhadores, seja para intervenção ou prevenção precoce ${ }^{7}$.

O exame admissional convencional, utilizado nas empresas, se inicia pela anamnese, abordando tanto o histórico clínico quanto o ocupacional. Tem a finalidade de ser - mais completa possível, pesquisando hábitos de vida, uso de medicamentos, doenças pregressas, cirurgias prévias e sinais e sintomas em cada aparelho ou sistema. Quando se aplica a CIF neste modelo, se consegue utilizar as categorias de funções e estruturas do corpo para realizar a anamnese e é possível categorizá-la em leve, moderada, grave, completa e não especificada, quando for identificado alguma alteração ${ }^{1,3}$.

De uma maneira suplementar, a CIF permite a inclusão das categorias de atividade, participação e fatores ambientais, o que, de forma superficial, não parecem estar relacionados com à aptidão do trabalhador para a função na empresa. No entanto, quando se identifica nesse trabalhador, dificuldades no âmbito social e se direciona para uma intervenção precoce, se está, possivelmente, evitando um acidente de trabalho ou um afastamento previdenciário prolongado, exemplos esses que geraria um grande ônus para a empresa.

Em um processo de contratação devese considerar todos os quesitos necessários para a execução das atividades profissionais com segurança e livres de problemas de desempenho. A CIF é uma ferramenta que descreve todos esses aspectos corroborando para um diagnóstico mais completo, que permita um acompanhamento amplo dos trabalhadores nos exames periódicos ${ }^{8}$.

O uso da CIF nos exames periódicos e de retorno ao trabalho, se consegue detectar precocemente as limitações do trabalhador, bem como o grau dessas dificuldades. Essa abordagem precoce possibilita a intervenção do médico do trabalho e da equipe do SESMT. É possível identificar, por exemplo, problemas ergonômicos, de fácil resolução, como uma mudança no ambiente de trabalho, evitando lesões no trabalhador ou um afastamento por doença.

A restrição laboral e o retorno ao trabalho estão entre os aspectos mais complexos das políticas de atenção à saúde do trabalhador. É necessário pensar a reabilitação profissional para além do que é feito no programa de reabilitação profissional da Previdência Social e da clássica reabilitação clínica oferecida em diferentes serviços de saúde, mas, também, considerála como um processo dinâmico de atendimento global do trabalhador que deve envolver as empresas no processo de prevenção, tratamento, reabilitação, readaptação e reinserção no trabalho ${ }^{9}$.

$\mathrm{O}$ afastamento do trabalho por tempo prolongado, segundo alguns autores (tempo acima de 3 meses, torna-se preditivo de dificultadores para o retorno ao trabalho, principalmente devido ao impacto psíquico do afastamento e do retorno, quando esses implicam em mudanças de posto de trabalho, de função e de status ${ }^{9}$.

Estudos mostram que os apoios e os relacionamentos, compreendidos na CIF como fatores ambientais, são colocados como aspectos facilitadores para o retorno ao trabalho. Considera-se que a relação dos profissionais da equipe com as chefias dos trabalhadores, a aceitação e o apoio dos colegas de trabalho, bem como da família, são considerados como fatores essenciais para o processo de reinserção no trabalho ${ }^{9}$. 
A CIF permite que qualquer indivíduo transite por uma linha imaginária no exercício de alguma atividade ou participação social, ora tendendo para a incapacidade, ora para a funcionalidade, na dependência não só do seu estado de saúde, de suas características pessoais e do que o meio lhe oferece ou deixa de oferecer ${ }^{7}$. Fazer com que o médico do trabalho utilize a CIF desde o exame admissional, nos exames periódicos e de retorno ao trabalho trariam diversas vantagens para $o$ trabalhador e para a empresa. A própria readaptação que o médico do trabalho pode fazer ou o enquadramento na Lei de Cotas podem ser solucionadas dentro da própria empresa, sem a necessidade de encaminhamento para a Previdência Social. Nesse sentido, a classificação ajuda a determinar graus de funcionalidade necessários para cada posto de trabalho e, ainda, fatores ambientais que possam garantir um desempenho livre de restrições. Um trabalhador, por exemplo, com determinada dificuldade de mobilidade será contratado para um posto de trabalho no qual tal domínio não seja exigido ou, caso necessite, facilitadores ambientais garantirão o desempenho pleno ${ }^{8}$.

Ações de promoção da saúde com mudanças de hábitos de vida, na alimentação, estímulo a atividades físicas podem contribuir na diminuição da prevalência de doenças crônicas não transmissíveis (DCNT), que em 2010 , foram a principal causa de mortalidade no Brasil $\left(73,9 \%\right.$ dos óbitos) ${ }^{10}$.

Quando olhamos o trabalhador como um todo, identificando suas comorbidades, que muitas vezes, não geram afastamentos, mesmo assim, diminuem sua produtividade, o que chamamos de presenteísmo. O custo de presenteísmo é "camuflado", porque o empregado está no trabalho, contudo sem produzir o esperado. Muitos empregados estão trabalhando sem se sentir bem e procuram exercer suas atividades, no entanto, suas condições de saúde acabam afetando o seu desempenho ${ }^{12}$.
Segundo dados da Previdência Social, em um estudo do ano de 2000 a 2011 as doenças motivadas por fatores de risco ergonômicos e a sobrecarga mental tem superado as causas traumáticas. As doenças do grupo $M$ e $F$ (riscos ergonômicos $e$ mentais) da CID, juntas alcançaram peso de $20,76 \%$ de todos os afastamentos, superando aquelas do grupo S-T (traumáticos) com $19,43 \%$ do total. (1 boletim). Dentre os 20 CIDs que mais afastam, 0 de maior prevalência foi M54 - dorsalgia (7,03\%), seguido por fratura de punho e mão (4.86\%), fratura de perna e tornozelo $(3,67 \%)$ e episódios depressivos (3,55\%). Há uma mudança no perfil produtivo do país, uma diminuição dos acidentes típicos de trabalho e um aumento das doenças relacionadas ao trabalho, sejam elas do sistema osteomuscular, como as relacionadas a saúde mental ${ }^{11,14}$.

De acordo com Alvarez (2020), todo médico sabe a CIF, mesmo que não tenha consciência desse saber. Todo médico sabe as repercussões que as doenças têm sobre as pessoas. Todo médico sabe as doenças pelo nome, sobrenome, tipo e subtipo. Aprendeu a classificá-las por códigos (CID) e, por que não, ampliar esse conhecimento e inserir a CIF como uma linguagem da doença e dos fatores relacionados que influenciam diretamente 0 estado da pessoa e uniformizarmos uma linguagem que permita comparações, avaliar evoluções e fornecer dados estatísticos? Todo médico é especialista em pessoas e faz uso da CIF diariamente, mesmo que não tenha consciência disso ${ }^{13}$.

Com a utilização da CIF, pode-se inferir que a incapacidade não é mera consequência de uma deficiência. A presença de barreiras à participação social, atitudinal, pode caracterizar um estado de incapacidade ${ }^{15}$. Por isso é importante a criação de programas focados nos aspectos citados para garantir a equidade ${ }^{15}$. 


\section{CONSIDERAÇÕES FINAIS}

A utilização da CIF nos exames médicos ocupacionais torna-se um aliado, tanto do médico do trabalho, quanto da empresa e do trabalhador. Isso porque, em todos os exames médicos ocupacionais pode ser feita a mesma abordagem utilizando as categorias da CIF. No exame admissional, os dados coletados servirião como uma base para 0 acompanhamento laboral do trabalhador, não substituindo o raciocínio clínico habitual do médico para a definição de aptidão. Nos exames periódicos e de retorno ao trabalho, ao identificar alterações nos indicadores, o médico do trabalho pode agir precocemente, na necessidade de acompanhamento com uma equipe multiprofissional, encaminhando para outra especialidade médica ou agindo em questões de gestão e organizacional que possam ser modificáveis. Nesse sentido, haverá uma atenção integral ao trabalhador, evitando afastamentos do trabalho, diminuindo acidentes, que causariam prejuízos para a empresa.

A saúde do trabalhador é um campo promissor e desafiador para a aplicação da $\mathrm{CIF}$. Introduzir as categorias aqui sugeridas nos exames ocupacionais, seria o primeiro passo para otimizar a atenção ao trabalhador. Considera-se essencial cuidar da saúde física e mental de quem mais precisa, e o qual nenhuma tecnologia avançada substituirá, o ser humano.

\section{REFERÊNCIAS BIBLIOGRÁFICAS:}

1. ORGANIZAÇÃO MUNDIAL DA SAÚDE. CIF. Classificação internacional de funcionalidade, incapacidade e saude. Lisboa; 2004.

2. Di Nubila H. Uma introdução à CIF - Classificação Internacional de Funcionalidade, Incapacidade e Saúde. Rev. bras. Saúde ocup., São Paulo, 35 (121): 122-123, 2010

3. ORGANIZAÇÃO MUNDIAL DA SAÚDE. CIF: Classificação Internacional de Funcionalidade, Incapacidade e Saúde. Tradução Centro Colaborador da Organização Mundial da Saúde para a Família de Classificações Internacionais. São Paulo: Edusp, 2003.

4. Di Nubila, H.B.V \& Buchalla, C.M. O papel das Classificações da OMS - CID e CIF nas definições de deficiência e incapacidade. Rev Bras Epidemiol 2008; 11(2): 324-35

5. Philereno DC, et al. QUALIFICAÇÃO DAS PESSOAS COM DEFICIÊNCIA PARA O MERCADO DE TRABALHO: UM ESTUDO DE CASO EM CAXIAS DO SUL - RS. ESTUDO \& DEBATE. 2015 Jan 01;22(1):160-179.

6. Teixeira J. Planejamento e Gestão do Programa de Controle Médico de Saúde Ocupacional (PCMSO): Medicina do Trabalho. $2^{\circ}$ edição. Atheneu, editor. São Paulo; 2016. 273 p.

7. Mendes R. Patologia do Trabalho. $3^{\circ}$ edição. Atheneu, editor. São Paulo; 2013. 2000, 2 vol. p.

8. Santana Cordeiro E. A CIF como norteadora da admissão, da inclusão e das indenizações. Revista Científica CIF Brasil. 2018;10(1):12-20.

9. Toldrá $\mathrm{R}$, et al. Facilitadores e barreiras para o retorno ao trabalho: a experiência de trabalhadores atendidos em um Centro - de Referência em Saúde do Trabalhador SP, Brasil. Revista Brasileira de Saúde Ocupacional. 2010 Mar 15;35(121):10-22. 
10. Epidemiol. Serv. Saúde v.21 n.4 Brasília dez. 2012.

11. Coordenação- Geral de Monitoramento Benefício por Incapacidade - CGMBI/DPSSO/SPS/MPS. Informe Especial por Ocasião do Dia Mundial em Homenagem às Vítimas de Acidente do Trabalho. 1ํㅡㄹ Boletim Quadrimestral sobre Benefícios por Incapacidade. 2014 Apr 25.

12. Zétola PR. Tratado de gestão em saúde do trabalhador. Belo Horizonte: Ergo; 2020. 856 p.

13. Alvarez M. Todo médico sabe CIF. www.saudeocupacional.org.br. 2020.

14. Simonelli AP, Camarotto JA, Spiridião Bravo E, Andrade de Gouveia Vilela R. Proposta de articulação entre abordagens metodológicas para melhoria do processo de reabilitação profissional. Revista Brasileira Saúde Ocupacional. 2010;35(121):64-73.

15. Munguba M, el al. DA INVISIBILIDADE À PARTICIPAÇÃO SOCIAL: PROMOÇÃO DA SAÚDE EM PESSOAS COM DEFICIÊNCIA. Rev Bras Promoç Saúde, Fortaleza, 28(4): 463-466, out./dez., 2015. 